The Egyptian Journal of Hospital Medicine (October 2019) Vol. 77 (2), Page 4956-4963

\title{
Impact of Temporal Lobe Epilepsy on Central Auditory Processing in Children Naema Mohammed Ismail ${ }^{1}$, Amany Ahmed Shalaby ${ }^{2}$, Ghada Saed Abdel Azim ${ }^{3}$, Eman Ismail Abd-Ellatif ${ }^{1}$ \\ ${ }^{1}$ Department of ENT, Audiology unit, Faculty of Medicine (for Girls), Al-Azhar University, ${ }^{2}$ Department of ENT, Audiology unit, Faculty of Medicine, Ain Shams University, ${ }^{3}$ Department of Neurology, Faculty of Medicine (for Girls), Al-Azhar University, Cairo, Egypt \\ * Corresponding author: Ghada Saed Abdel Azim, Email: ghada_saed2006@yahoo.com
}

\begin{abstract}
Background: Anatomical and functional integrity of the auditory pathways, from peripheral to central structures, is essential for proper processing of auditory stimuli and a satisfactory development of language, speech, and learning. Neurological disorders, such as epilepsy, are one of the possible causes of central auditory disorders.

Objective: The aim of the current work was to study the effect of temporal lobe epilepsy on auditory temporal resolution ability in children.

Patients and methods: Thirty children suffering from temporal lobe epilepsy, with age ranged from 12 to 16 years, were included in this study. Also, 10 apparently healthy children with age and gender matched with the study group were taken as a control group. All children were subjected to full history taking, clinical examination, cognitive and basic audiological evaluation. Also, questionnaire for central auditory processing disorders in children was applied and assessment of temporal resolution ability using Gaps In Noise (GIN) test.

Results: Children with temporal lobe epilepsy had significantly increased gap detection threshold (GDTh) values, and lower percentage of correct gap detection than control group. $86.7 \%$ of epileptic children demonstrated abnormal scores for approximate GIN threshold (gap detection threshold above $6 \mathrm{msec}$, with sensitivity and specificity of $80 \%$ and $100 \%$ for right ear and $86 \%$ and $100 \%$ for left ear.

Conclusion: Temporal resolution deficit is a comorbidity in children with temporal lobe epilepsy and is increased with longer duration of epilepsy. Impairment was not affected by age, gender, medications or duration of treatment. Also, there was no significant difference between both ears in GIN test, reflecting no laterality effect.
\end{abstract}

Keywords: Temporal lope Epilepsy; Auditory Temporal Resolution; Gaps in Noise Test.

\section{INTRODUCTION}

Central auditory processing refers to perceptual processing of auditory information in the central nervous system (CNS). If there is any defect in central auditory processing, the sound will not be recognized properly, resulting in information error ${ }^{(1)}$.

Central Auditory Processing Disorder (CAPD) is defined as a deficit in neural processing of auditory information in the central nervous system. According to American Speech-Language Hearing Association (ASHA), CAPD may coexist with other disorders and may be associated with difficulties in higher order language, learning, and communication functions ${ }^{(2)}$.

Epilepsy is a brain disorder characterized by recurrent and unpredictable interruptions of normal brain function, called epileptic seizures. Epileptic seizure is a transient occurrence of signs and/or symptoms due to abnormal excessive or synchronous neuronal activity in the brain ${ }^{(3)}$.

Temporal Lobe Epilepsy (TLE) is one of the most common types of epilepsy in childhood, which may reflect the vulnerability of the temporal lobe to a variety of cerebral insults ${ }^{(4)}$.

The occurrence of epileptic seizures in the developing brain may impair neurophysiological maturation of neural networks involved in the acquisition of cognitive skills that are fundamental to the learning process. The dysfunction may be due to an abnormal cortical processing, neuronal organization, and functional specialization of the brain ${ }^{(5)}$.

The topography of spikes in TLE, with epileptic activity in centrotemporoparietal, perisylvian, temporoparietal, and temporal regions, suggests that they may originate near auditory and language cortices. Electrical discharges are close to the final point of the auditory pathway in this type of epilepsy ${ }^{(6)}$.

It has been recognized that some patients with epilepsy, especially TLE, have speech recognition impairments or speech processing difficulty, (7) even though they have normal auditory function. This suggests that patients with TLE may have functional deficits in central auditory processing (CAP) ${ }^{(2)}$.

Temporal auditory processing (TAP) is defined as the perception of the temporal envelope or the alteration of durational characteristics of a sound within a restricted or defined time interval. Some authors argued that TAP may be the underlying component of many auditory processing abilities, including the processing of both verbal and nonverbal acoustic signals such as speech perception in quiet and in background noise, localization, discrimination, binaural integration, and binaural separation $^{(8,9,10)}$.

Among temporal auditory skills that can be assessed by behavioral tests, there are temporal resolution and temporal ordering. Such abilities require an accurate 
processing of the sound time, structure of the signal, time and order of occurrence. Those abilities help to identifying small phonetic elements in speech, important cues that help speech perception. Difficulties found in these skills suggest interference in normal speech perception and phonemes recognition ${ }^{(11)}$.

Temporal resolution ability includes the shortest duration of time which an individual can discriminate between two signals. It can be assessed using a variety of different procedures and Gaps-in-Noise (GIN) test is described as a procedure with good specificity ${ }^{(12)}$.

The aim of the current work was to study the effect of temporal lobe epilepsy on auditory temporal resolution ability in children.

\section{SUBJECTS AND METHODS}

This case control comparative study included a total of 40 Egyptian Children, 30 of them were suffering from temporal lobe epilepsy, attending at Outpatient Neurology Clinic, Al-Zahraa University Hospital. This study was conducted between November 2016 to August 2017.

\section{Ethical approval:}

\section{Approval of the ethical committee was obtained.}

The assessment started after the parents read the Information Letter about the study goals and signed the Informed Consent Form to participate in the study.

The included subjects were divided into two groups; Group 1 (epileptic) consisted of 30 Egyptian Children of both gender, aged 12 to 16 years, and had been neurologically diagnosed with temporal lobe epilepsy according to criteria of International League Against Epilepsy (ILAE) 2010 classification of seizure types ${ }^{(13)}$ on the basis of clinical history of seizure and EEG. They were neurologically free except for temporal lobe epilepsy, with normal brain MRI, with average or above average intelligence and normal hearing sensitivity not exceeding $20 \mathrm{~dB}$ in the frequency range $250-8000 \mathrm{~Hz}$ and normal middle ear functions as evidenced by otological examination, tympanometry and acoustic reflex threshold, Group 2 (control) included a group of 10 normal Egyptian children who were age and gender matched with the study group. They had no history suggestive of diseases involving central nervous system (CNS), no hearing complaints or difficulties reported by parents, no history of delay or abnormality in language and learning, with average or above average intelligence, normal hearing sensitivity, and normal middle ear functions examination.

Both the study groups were subjected to full history taking including perinatal, developmental history, past medical history for otological and neurological diseases, history of epilepsy as regard duration of illness, type of medication and duration of treatment.

Also, they underwent assessment of cognitive abilities using Stanford Binet test Arabic version (SB-5)
(14) and detecting central auditory abilities by questionnaire for central auditory processing disorders in children . ${ }^{(15)}$ Then Gaps In Noise (GIN) test ${ }^{(9)}$ was administered to test for temporal resolution ability. In the GIN test, subjects should identify when gaps occur in a noise stimulus. The noise is a Gaussian-distributed white noise generated with a sampling rate of $44.1 \mathrm{kHz}$. Noise segments are $6 \mathrm{sec}$ in duration, within which 0 to 3 gaps may be embedded. The gap durations presented are 2, 3, $4,5,6,8,10,12,15$, and $20 \mathrm{~ms}$. In each GIN list, the individual gap durations are presented 6 times each in random locations across the various trials, for a total of 60 gaps. Four lists are available for testing which allows for test-retest comparisons. Ten practice items have preceded the administration of the test items. The study was approved from the ethical committee of Faculty of Medicine, Al-Azhar University.

\section{Statistical Analysis}

The collected data was revised, coded, tabulated and introduced to a PC using Statistical Package for Social Science (SPSS 20). Data was presented and suitable analysis was done according to the type of data obtained for each parameter.

\section{I - Descriptive statistics:}

1. Mean, Standard deviation $( \pm \mathrm{SD})$ for numerical data.

2. Frequency and percentage of non-numerical data.

\section{II - Analytical statistics:}

1. Student $\mathbf{t}$ test: to assess the statistical significance of the difference between two study group means.

2. Fisher's exact test: to examine the relationship between two qualitative variables when the expected count is less than 5 in more than $20 \%$ of cells

3. Correlation analysis (using Pearson's method): To assess the strength of association between two quantitative variables. The correlation coefficient denoted symbolically " $r$ " defines the strength (magnitude) and direction (positive or negative) of the linear relationship between two variables. Probability (P-value) $<0.05$ was considered significant, (P-value) $<0.01$ was considered highly significant, and (P-value) $>0.05$ was considered insignificant.

\section{RESULTS}

\section{Characteristics of the study population:}

This study included 30 children with temporal lobe epilepsy, and 10 age and sex matched healthy control children. The mean age in the epileptic group was $13.8 \pm 1.5$ years and in the control group it was $14.3 \pm 1.2$ years. In the epileptic group they were 14 males (46.7\%) and 16 females $(53.3 \%)$, and in the control group there were 5 males (50\%) and 5 females (50\%), with no statistically significant difference between both groups as regard age and gender. 
The mean duration of illness in the epileptic children was $3.8 \pm 2.9$ years, and the mean duration of treatment was $2.3 \pm 1.9$ years.

Results of Central Auditory Processing questionnaire: Compared to controls, the epileptic children had significantly reduced scores as regard sustained and selective attention, audio-visual integration, memory, behavior and scholastic achievement.

However, no statistically significant difference between the epileptic and the controls as regard localization and identification or language abilities (table: 1).

\section{Results of GIN test:}

Children with temporal lobe epilepsy had significantly increased gap detection threshold (GDTh) values and lower percentages of correct gap detection than control group, indicating significant impairments in temporal processing in this clinical population; and no significant difference was found in the GDTh or the percentage of correct responses between ears.(table 2, fig: 1,2 )

There was no statistically significant difference between both genders in GIN test measures.
Also, Gap detection threshold of 6 msec. was the cut off threshold for abnormality with sensitivity and specificity of $80 \%$ and $100 \%$ respectively for right ear and with sensitivity and specificity of $86 \%$ and $100 \%$ respectively for left ear.(fig:3,4)

It was found that 26 out of 30 patients $(86.7 \%)$ of epileptic children demonstrated abnormal scores for approximate GIN threshold (GDTh) with reference to cut off point $>6$ msec. (Table 3 ).

Effect of age, duration of disease, and duration of treatment on GIN test measures:

There was significant negative correlation between (GIN total correct score - total GIN Score \%) and duration of disease. Also, there was significant positive correlation between Approx. GIN Threshold /msec. and duration of disease. However, there was no significant correlation between (Age - Duration of treatment) \& GIN test measures. (Table: 4)

Table (1): Comparison between control and study groups as regards central auditory processing questionnaire.

\begin{tabular}{|l|l|l|l|l|l|l||}
\hline \multirow{2}{*}{ Auditory Behavior Questionnaire } & \multicolumn{2}{l|l}{ Control group } & \multicolumn{2}{l|}{ Study group } & \multicolumn{2}{l|}{ t test } \\
\cline { 2 - 8 } & Mean & SD & Mean & SD & \multicolumn{4}{l|}{ p value } & sig. \\
\hline \hline Localization and identification & 100.0 & 0.0 & 100.0 & 0.0 & \multicolumn{4}{|l|}{$\mid$} \\
\hline Sustained and selective attention & 95.0 & \pm 10.5 & 45.8 & \pm 21.9 & $<0.001$ & $\mathrm{~S}$ \\
\hline Audio-visual integration & 100.0 & \pm 0.0 & 86.7 & \pm 26.0 & 0.009 & $\mathrm{~S}$ \\
\hline Memory & 92.5 & \pm 12.1 & 60.0 & \pm 25.9 & $<0.001$ & $\mathrm{~S}$ \\
\hline Scholastic achievement & 95.0 & \pm 10.5 & 78.3 & \pm 14.3 & 0.002 & $\mathrm{~S}$ \\
\hline Language & 100.0 & \pm 0.0 & 98.3 & \pm 6.3 & 0.415 & $\mathrm{NS}$ \\
\hline Behavior & 6.0 & \pm 9.7 & 27.3 & \pm 11.1 & $<0.001$ & $\mathrm{~S}$ \\
\hline
\end{tabular}

Table (2): Comparison between control and study groups as regards GIN(Gaps in noise) test measures.

\begin{tabular}{||l|l|l|l|l|l|l|l||}
\hline \multicolumn{2}{|c|}{} & \multicolumn{2}{l|}{ Control group } & \multicolumn{2}{l|}{ Study group } & \multicolumn{1}{l|}{ test } \\
\cline { 3 - 8 } & Mean & SD & Mean & SD & p value & sig. \\
\hline \hline \multirow{2}{*}{ GIN total correct score ( /60) } & RT & 44.6 & \pm 2.6 & 29.5 & \pm 4.4 & $<0.001$ & S \\
\cline { 2 - 8 } & LT & 43.8 & \pm 2.8 & 30.0 & \pm 4.4 & $<0.001$ & S \\
\hline \multirow{3}{*}{ Total GIN Score (\%) } & RT & 74.3 & \pm 4.3 & 49.2 & \pm 7.3 & $<0.001$ & S \\
\cline { 2 - 8 } & LT & 73.0 & \pm 4.6 & 49.9 & \pm 7.4 & $<0.001$ & S \\
\hline \multirow{2}{*}{ Approx. GIN Threshold (msec.) } & RT & 4.8 & \pm .8 & 8.8 & \pm 1.7 & $<0.001$ & S \\
\cline { 2 - 8 } & LT & 4.8 & \pm .8 & 8.5 & \pm 1.6 & $<0.001$ & S \\
\hline
\end{tabular}

Table (3) Number and percent of patient (epileptic children) with abnormal approximate GIN threshold.

\begin{tabular}{|l|c|c||}
\hline & Number & Percent \\
\hline \hline Pt. with abnormal Approx. GIN threshold /msec. & 26 & $86.7 \%$ \\
\hline Pt. with normal Approx. GIN threshold /msec. & 4 & $13.3 \%$ \\
\hline \hline
\end{tabular}


Table (4): Effect of age, duration of disease and duration of treatment on GIN Test measures in study group.

\begin{tabular}{|c|c|c|c|c|c|c|c|}
\hline & & \multicolumn{2}{|l|}{ Age } & \multicolumn{2}{|c|}{$\begin{array}{l}\text { Duration of disease / } \\
\text { year }\end{array}$} & \multicolumn{2}{|c|}{ Duration of treatment } \\
\hline & & $\mathbf{R}$ & p value & $\mathbf{R}$ & p value & $\mathbf{R}$ & p value \\
\hline \multirow{2}{*}{ GIN total correct score } & RT & -0.033 & 0.861 & -0.419 & $0.021 *$ & -0.080 & 0.673 \\
\hline & LT & -0.006 & 0.975 & -0.563 & $0.001 *$ & -0.185 & 0.327 \\
\hline \multirow{2}{*}{ Total GIN Score\% } & RT & -0.034 & 0.859 & -0.418 & $0.022 *$ & -0.079 & 0.677 \\
\hline & LT & -0.005 & 0.978 & -0.564 & $0.001 *$ & -0.186 & 0.325 \\
\hline \multirow{2}{*}{$\begin{array}{l}\text { Approx. GIN Threshold } \\
\text { msec. }\end{array}$} & RT & 0.107 & 0.573 & 0.384 & $0.036^{*}$ & 0.162 & 0.391 \\
\hline & LT & 0.262 & 0.161 & 0.587 & $0.001 *$ & 0.151 & 0.427 \\
\hline
\end{tabular}

*significant correlation

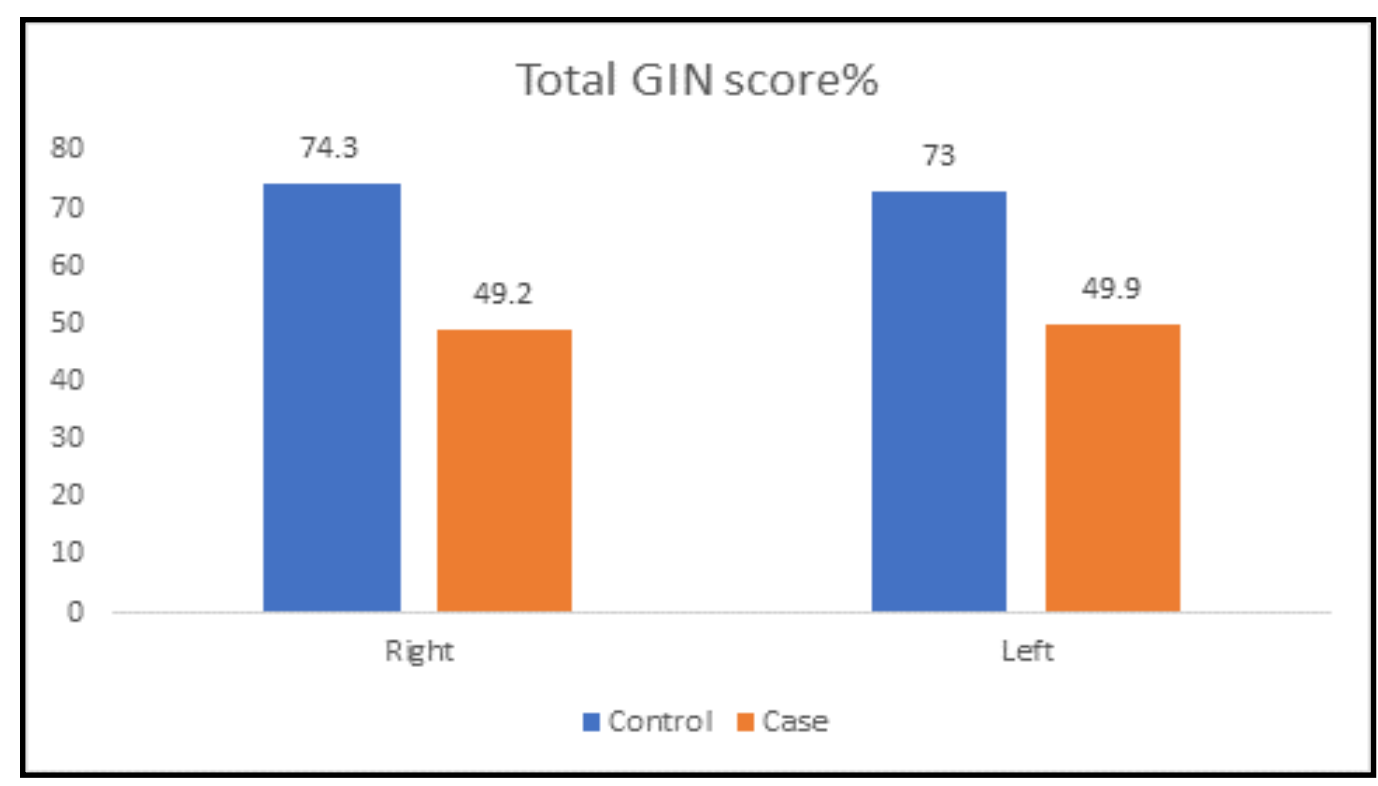

Figure (1): $\quad$ Comparison between control and study groups as regards Total GIN Score (\%).

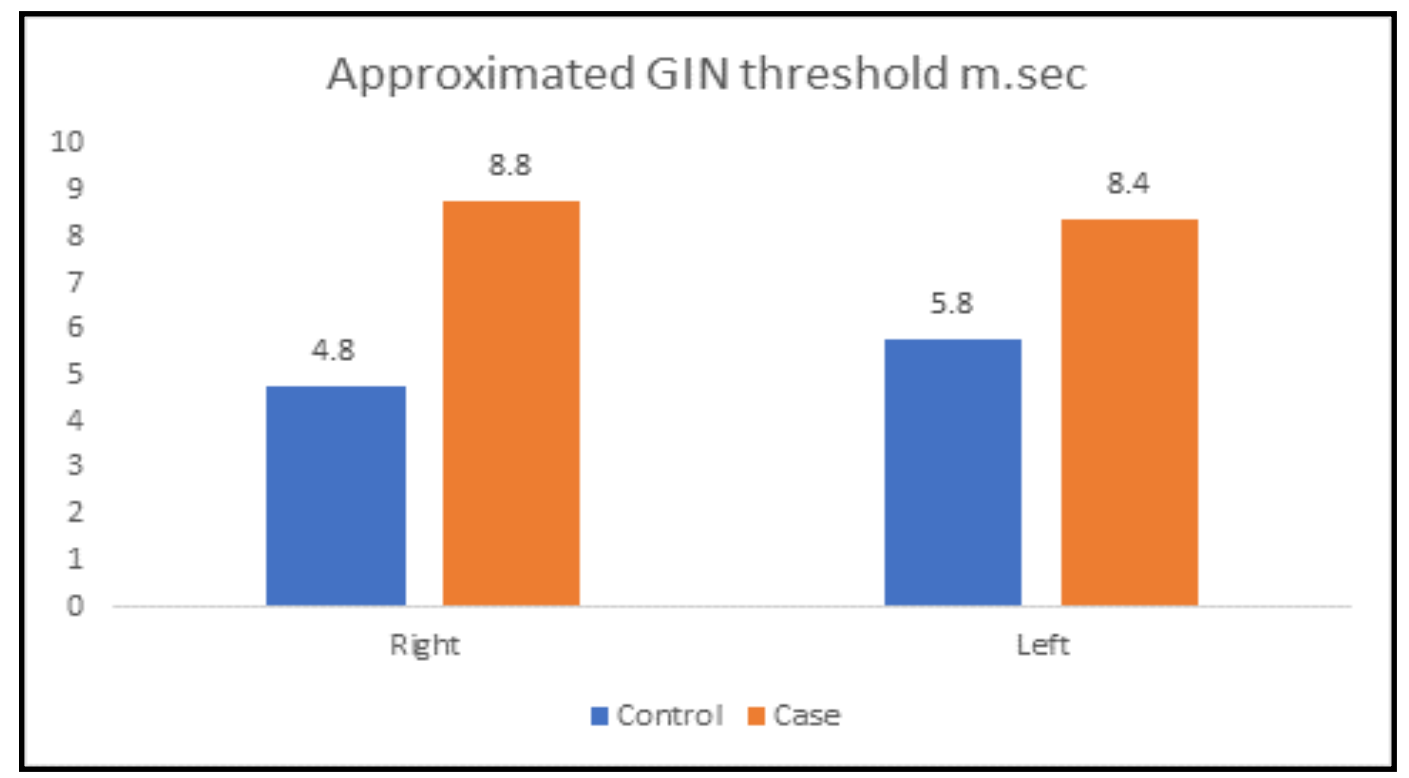

Figure (2): Comparison between control and study groups as regards Approx. GIN Threshold (msec.) 


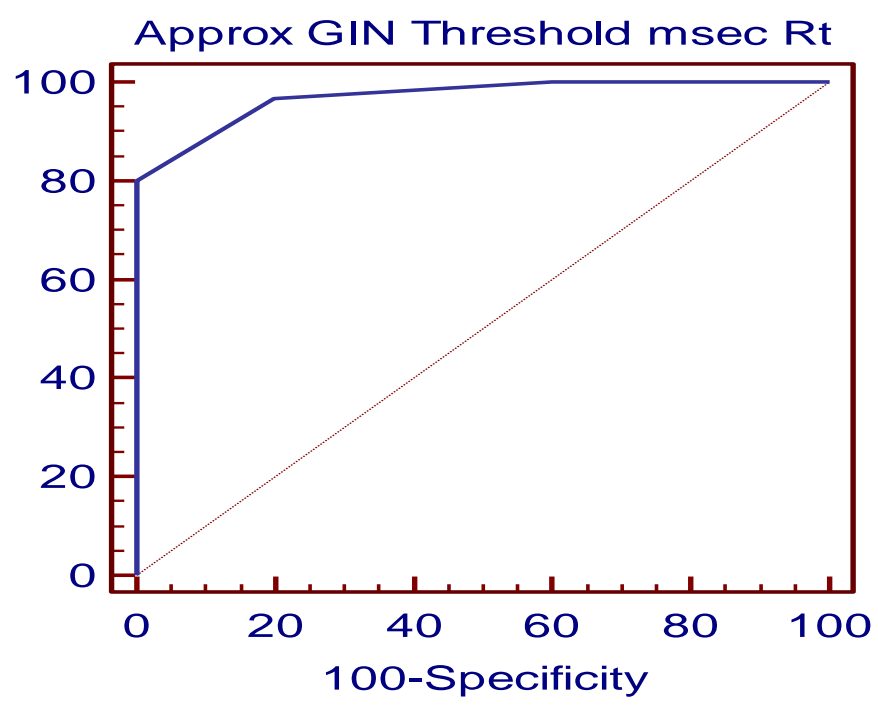

\begin{tabular}{|l|l|l|l|l|l|}
\hline AUC & 95\% CI & p value & Cut off point & sensitivity & Specificity \\
\hline 0.97 & $0.861-0.999$ & 0.001 & $>6$ & 80 & 100 \\
\hline
\end{tabular}

AUC: Area under the curve

CI: confidence interval

Figure (3): Receiver operating characteristic (ROC) curve for the gaps-in-noise test threshold (Approx. GIN Threshold /msec.) of right ear.

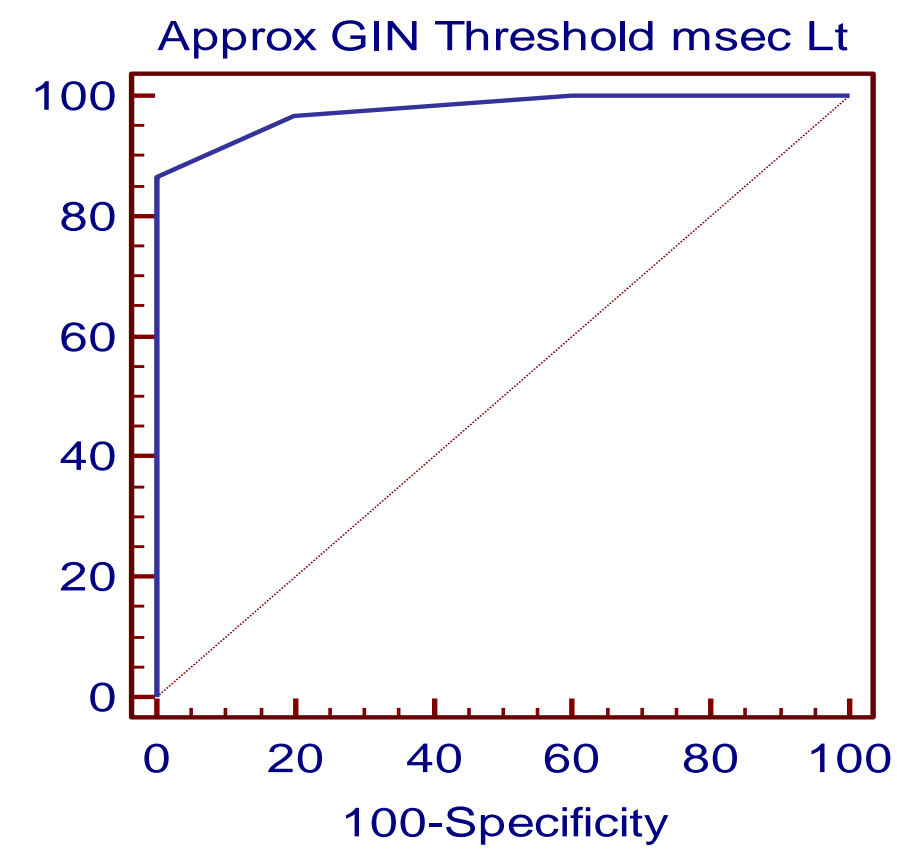

\begin{tabular}{|l|l|l|l|l|l|}
\hline AUC & 95\% CI & p value & Cut off point & sensitivity & Specificity \\
\hline 0.98 & $0.871-1$ & 0.001 & $>6$ & 86.7 & 100 \\
\hline
\end{tabular}

AUC: Area under the curve

CI: confidence interval

Figure (4): Receiver operating characteristic (ROC) curve for the gaps-in-noise test threshold (Approx. GIN Threshold /msec.) of left ear. 


\section{DISCUSSION}

Temporal lobe epilepsy (TLE) is the most common type of focal epilepsy. Previous research has shown that patients with TLE exhibit decreased performance in listening to speech sounds and deficits in the cortical processing of auditory information ${ }^{(7)}$.

In the present study, central auditory processing questionnaire was applied and reflected the presence of CAPD in epileptic children of study group in all abilities except for localization and language abilities. Not affected language ability could be attributed to the age of study group children (12-16 years) which was beyond the age of language development so abnormal epileptic electrical discharges didn't affect this ability. Language is rooted in early cognitive development ${ }^{(16)}$. It may be argued that there is a critical period during childhood when active language development occurs within a limited time frame. This is also known as a sensitive period, when circuits are being established and solidifying their connections among language structures. This age is believed to end around age 6 or 7 years old $\left({ }^{17)}\right.$.

As regard localization, there was no difference between control and study groups. This could be attributed to that the structures responsible for sound location are the superior olivary complex and the inferior colliculus, located at the brain stem ${ }^{(1)}$. Moreover, there are reports that any loss in the sound source direction discrimination assessed in a free field, suggests problems at the brain stem level. Thus, in patients with temporal lobe epilepsy, it would not be expected to see alterations in the Sound Localization ${ }^{(18)}$. This was in agreement with Meneguello et al. who found that epileptic patient had a similar performance to those age matching controls, as to their sound source direction discrimination (sound source location $)^{(18)}$.

As regard attention and selective attention, the results are partially in agreement with Hudson et al. who found that patients with TLE demonstrate marked deficits in selective attention, divided attention, and set shifting in comparison to controls. In contrast, sustained attention is less compromised and the capacity to allocate cognitive resources appears to be normal in patients with TLE ${ }^{(19)}$. Patients with (TLE) demonstrate impairments in a range of cognitive domains, including memory, language, and visuospatial functions. In addition, across different studies, deficits on tests of attentional control including the Wisconsin Card Sorting Test ${ }^{(20,21)}$, the Stroop ${ }^{(22)}$, and Trail Making Test (TMT) ${ }^{(23)}$ have been widely reported. Although the control of attention has a long-standing association with frontal lobe functioning, these studies have led to the hypothesis that attentional control may be modulated by the hippocampus and related medial temporal lobe structures. Alternatively, it may be postulated that impaired attentional control in TLE arises from abnormal discharges propagating from nociferous epileptogenic medial temporal lobe structures to otherwise healthy frontal regions ${ }^{(24)}$.

Also, the results corroborate with studies that have reported specific learning disabilities and decreased academic performance in children with temporal lobe epilepsy (TLE), which were demonstrated by tests and also by parents and teachers statement of academic underachievement ${ }^{(25,26)}$.

In the present study, children with temporal lobe epilepsy had significantly increased gap detection threshold (GDTh) values and lower percentages of total GIN scores than control group. This indicated significant impairments in temporal processing in this clinical population.

Poor GIN test performance of epileptic children in the present study could be explained by hypothesis that electrical discharges in these areas may result in impairment of cortical auditory function. The auditory function may be altered if there is a maturational deficit caused by epileptic activity in this brain area $\left({ }^{27)}\right.$.

As regard the effect of ear on GIN test results, it was obvious that there was no significant difference in the GDTh or the percentage of correct responses between ears.

This lack of laterality is probably related to the fact that the response to the GIN test involves the whole cortex, and not only its contralateral pathways ${ }^{(9)}$. Also, this could be attributed to the fact that maturation of the auditory system proceeded at the same rate for both the left and right aspects of the auditory system with respect to temporal resolution (28). In addition, contralateral seizure propagation in patients with temporal lobe epilepsy has also been reported, indicating that the condition may affect both sides ${ }^{(29)}$.

These findings are consistent with previous studies. Boscariol et al. investigated temporal auditory processing in children with temporal lobe epilepsy using GIN test. They observed a significant difference in auditory processing skills with worse performance in the group with epilepsy. Also, they analyzed the possible difference between right and left ears for GIN test and they found no significant difference between both ears ${ }^{(5)}$.

In addition, Amaral et al. examined temporal auditory processing of a sample of 13 children diagnosed with benign epilepsy of childhood with centrotemporal spikes (BECTS) using GIN test and found that 12 of 13 children $(92.3 \%)$ demonstrated abnormal results for gap detection threshold (above $6 \mathrm{~ms}$ ). Also no significant difference was found in performance of right and left ears (11). 
However, there is general agreement among researchers that epileptic patient are not able to process auditory information efficiently. Many other studies also performed on patients with temporal lobe epilepsy showed poor performance on different auditory processing abilities (temporal ordering, dichotic listening and processing of rapid auditory information) (18, 30, 31, 32, $33,34)$

Sensitivity and specificity were calculated based on the performance of subjects in the present study. ROC (receiver operating characteristic) curves indicated that $>$ 6 msec. is a cut-off for gap detection threshold. At this cut-off, a sensitivity and a specificity of $80 \%$ and $100 \%$, for right ear while a sensitivity and a specificity of $86 \%$ and $100 \%$, for left ear were noted. These findings indicated that the GIN test shows good efficiency in detecting auditory temporal processing lesion in children with temporal lobe epilepsy.

This was in agreement with Rabelo et al. who stated a cut off of $6.8 \mathrm{msec}$. with a sensitivity and specificity $68 \%$ and $98 \%$ respectively ${ }^{(35)}$. Moreover, Shinn et al. reported a sensitivity and specificity of GIN test $67 \%$ and $94 \%$ respectively for central auditory nervous system (CANS) lesion and a sensitivity of $80 \%$ for cortical lesion only. They explained this finding on bases that GIN is mediated at the level of cortex ${ }^{(28)}$.

According to the present GIN cut off ( $6 \mathrm{msec}$.), it was found that $86.7 \%$ of study group showed abnormality. This finding approximated the finding of Amaral et al, who found $92.3 \%$ of the study group demonstrated abnormal results for gap detection threshold (11).

Findings of the present study showed significant negative correlation between (GIN total correct score total GIN Score \%) and duration of disease. Also, there was significant positive correlation between Approx. GIN Threshold /msec. and duration of disease. These results indicate that longer duration of epilepsy associated with lower scores on GIN test measures.

Although the exact mechanism underlying the association between longer duration of epilepsy and CAP impairment is uncertain, these findings suggest a secondary functional and structural decline of the temporal lobe with increasing duration of disease as a progressive process in TLE.

The present study showed that there was no significant difference in GIN test measures between both genders. This result agreed with Han et al. who indicated no significant differences between both genders in CAP ability of patient diagnosed with temporal lobe epilepsy (34).

Also, there was no significant correlation between GIN test measures and age. This result agreed with
Tawfik et al. ${ }^{(30)}$, who reported no improvement in GIN test measures with age. Also, this result was in agreement with Shinn et al. ${ }^{(28)}$, who reported that no statistically significant difference was found between different age subgroups (7-18 years old) in GIN approximate thresholds. In the present study, there was insignificant correlation between GIN test measures and duration of receiving treatment. Also there was no statistically significant difference between different classes of medical treatment in GIN test measures. This was in agreement with Bakr et al..$^{(31)}$, who found that subjects with TLE showed no statistically significant difference in any of the psychophysical tests performed in his study (LPF, PPS and SSW) when classes receiving different types of therapy were compared. This also agreed with Han et al. ${ }^{(34)}$, who reported no statistically significant difference between dichotic tests in patients on multiple antiepileptic drugs (AEDs) therapy compared with patient taking one or two AEDs.

In conclusion, temporal resolution deficit is a comorbidity in children with temporal lobe epilepsy, which increased in severity with longer duration of the disease. GIN test appears to be a reliable and valid measure for assessment and early identification of temporal resolution deficits in temporal lobe epilepsy in children.

This study has some limitations. Because of the reduced number of subjects, some characteristics of seizure that can act as major generators of cognitive disorders were not explored, such as age at onset of seizures, lateralization of seizure, seizure frequency and duration.

Further studies with larger sample size are needed to elucidate the effect of the previous factors and different antiepileptic drugs on central auditory processing deficits in children with temporal lobe epilepsy.

\section{REFERENCES}

1. Bellis T(2003): Assessment \& management of central auditory processing disorders in the educational setting: from science to practice. Singular Audiology Text. 2nd ed. Clifton Park, NY: Thomson Delmar Learning.

2. American Speech-Language-Hearing Association(2005): Central auditory processing disorders [technical report]. Available at: http://www.asha. org/docs/html/TR200500043.html.

3. Blume W, Luders H, Mizrahi E, Tassinari C, van Emde Boas W and Engel J (2001):Glossary of descriptive terminology for ictal semiology: report of the ILAE Task Force on Classification and Terminology. Epilepsia, 42: 1212-8.

4. Aicardi J (1986): Epilepsies with affective--psychic manifestations and complex partial seizures. In: Epilepsies in children. New York: Raven Press. 
5. Boscariol M, Raquel L, Amaral M, Lunardi L, Carla G and Marilisa M (2015): Language and central temporal auditory processing in childhood epilepsies. Elsevier.

6. Rocha C, Miziara C, Manreza M and Schochat E (2010): Electrophysiological and auditory behavioral evaluation of individuals with left temporal lobe epilepsy. Arq. Neuropsiquiatr., 68: 18-24.

7. Boatman D, Lesser R, Crone N, Krauss G, Lenz F , Miglioretti D (2006): Speech recognition impairments in patients with intractable right temporal lobe epilepsy. Epilepsia , 47:1397-401.

8. Shinn JB and Musiek FE (2003): "Temporal processing: the basics," The Hearing Journal, 56:52.

9. Musiek FE, Shinn JB, Jirsa R, Bamiou D, Baran JA, Zaida E (2005) :GIN (Gaps-In-Noise) test performance in subjects with confirmed central auditory nervous system involvement. Ear and Hearing, 26 (6): 608-618,

10. Samelli A G and Schochat E (2008): The gaps-in-noise test: gap detection thresholds in normal-hearing young adults.International Journal of Audiology, 47: 238-245,

11. Amaral M, Casali R, Boscariol M, Lunardi L, Guerreiro $M$ and Colella-Santos M (2005): Temporal Auditory Processing and Phonological Awareness in Children with Benign Epilepsy with Centrotemporal Spikes. Biomed Research International.,25:6340.

12. Chermak G D and Lee J (2005): Comparison of children's performance on four tests of temporal resolution. Journal of the American Academy of Audiology, 16, : 554-563.

13. Berg A, Berkovic S, Brodie M et al. (2010): Revised terminology and concepts for organization of seizures and epilepsies: report of the ILAE Commission on Classification and Terminology. Epilepsia, 51:676-685.

14. Hanoura M(2002): Stanford Binet Intelligence test: Arabic version. Cairo: Anglo Press.

15. Tawfik $S$ and Shalaby A (1995): Development and standardization of Arabic central test battery for children. Proceeding of XX11l world congress Of the International Association of Logopedics and phoniatric,; Pp.416-419.

16. Kaderavek J N(2011): Language disorders in children: Fundamental concepts of assessment and intervention. New Jersey: Pearson Educ. Inc.

17. Monjauze C, Tuller L, Hommet $C$, Barthez $M A$ and Khomsi A (2005): Language in benign childhood epilepsy with Centro-temporal spikes abbreviated form: Rolandic epilepsy and language. Brain and Language, 92: 300-308.

18. Meneguello $J$, Leonhardt $F$ and Pereira $L$ (2006): Auditory processing in patients with temporal lobe epilepsy. Brazilian Journal of Otorhinolaryngology, 72(4):496-504.

19. Hudson JM, Flowers KA, Walster KL(2014) :Attentional control in patients with temporal lobe epilepsy, The British Psychological Society, Journal of Neuropsychology, 8:140 146
20. Corcoran $R$ and Upton D (1993): A role for the hippocampus in card sorting. Cortex, 29: 293-304.

21. Hermann B and Seidenberg M (1995): Executive system dysfunction in temporal lobe epilepsy: Effects of nociferous cortex versus hippocampal pathology. Journal of Clinical and Experimental Neuropsychology, 17: 809-819.

22. McDonald CR, Delis D C, Norman M A, Wetter S R, Tecoma E S and Iragui, V J (2005): Response inhibition and set shifting in patients with frontal lobe epilepsy or temporal lobe epilepsy. Epilepsy and Behaviour, 7:438-446.

23. Piazzini A, Turner K, Chifari R, Morabito A, Canger $R$ and Canevini M P (2006): Attention and psychomotor speed decline in patients with temporal lobe epilepsy: A longitudinal study. Epilepsy Research; 72, 89-96.

24. Catenoix H, Magnin M, Guenot $M$, Mauguiere $F$ and Ryvlin P (2005): Hippocampal-orbitofrontal connectivity in human: An electrical stimulation study. Clinical Neurophysiology,116: 1779- 1784.

25. Piccinelli P, Borgatti R, Aldini A et al. (2008): Academic performance in children with rolandic epilepsy. Developmental Medicine \& Child Neurology, 50(5):353356.

26. Miziara C, de Manreza M, Mansur L et al. (2012): Impact of benign childhood epilepsy with centrotemporal spikes (BECTS) on school performance. Seizure, 21(2):87-91.

27. Lundberg S, Frylmark A, Eeg-Olofsson O (2005): Children with rolandic epilepsy have abnormalities of oromotor and dichotic listening performance. Developmental medicine and child neurology, 47:603-8.

28. Shinn J, Chermak G, Musiek F (2009): GIN (Gaps-InNoise) Performance in the Pediatric Population. Journal of the American Academy of Audiology, 20:229-238.

29. Adam C, Hasboun D, Clemenceau S, Dupont S, Baulac M, Hazemann P (2004): Fast contralateral propagation of after discharges induced by stimulation of medial temporal lobe. Journal of Clinical Neurophysiology 21((6)):399-403.

30. Tawfik $S$ (1991): central auditory tests in epileptics. Ain Shams Medical Journal, 4: 27-42.

31. Bakr M, Tawfik S, Saad M, Farrag A, Hassan A(1994) : Central auditory tests in epileptic patients. M D Thesis, Faculty of medicine, Assiut University .

32. Ehrlé N, Samson S, Baulac M (2001): Processing of rapid auditory information in epilepticpatients with left temporal lobe damage. Neuropsychologia, 39:525-31.

33. Ortiz K, Pereira L, Borges A, Vilanova L (2002): Staggered spondaic word test in epileptic patients. São Paulo Medical Journal, 120:185-8.

34. Han M, Kang J, Lee E, Lee J, Bae J, Chung J (2011): Central auditory processing impairment in patients with temporal lobe epilepsy. Elsevier.

35. Rabelo C, Weihing J , Schochat E(2015): Temporal resolution in individuals with neurological disorders. Clinics (Sao Paulo), 70(9): 606-611. 